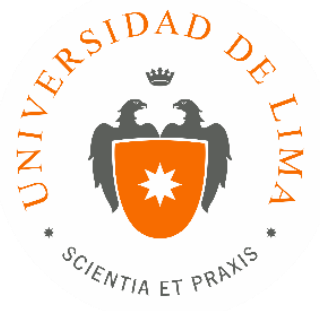

\title{
DISEÑO DE UN PROGRAMA DE EDUCACIÓN EMOCIONAL PARA ALUMNOS DE SEXTO GRADO EN UNA INSTITUCIÓN EDUCATIVA PRIVADA DE LIMA METROPOLITANA
}

Trabajo de suficiencia profesional para optar el título profesional de Licenciado en Psicología

Daniela Alessandra Coppo Escobedo

Código 20120353

Lima - Perú

Febrero de 2019 


\section{DISEÑO DE UN PROGRAMA DE EDUCACIÓN EMOCIONAL PARA ALUMNOS DE SEXTO GRADO EN UNA INSTITUCIÓN EDUCATIVA PRIVADA DE LIMA METROPOLITANA}




\section{TABLA DE CONTENIDO}

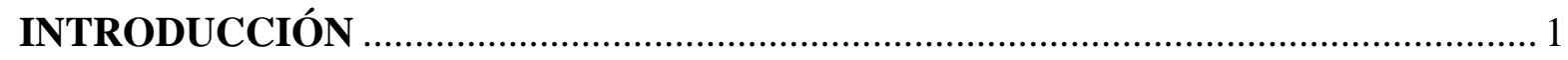

CAPÍTULO I: IDENTIFICACIÓN DEL PROBLEMA .............................................. 2

CAPÍTULO II: DESCRIPCIÓN DE LAS ACTIVIDADES Y TAREAS REALIZADAS

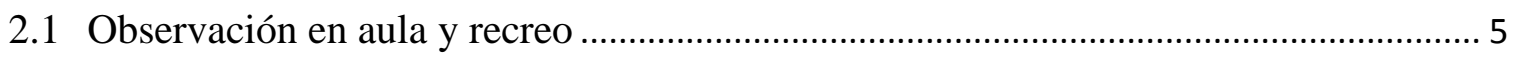

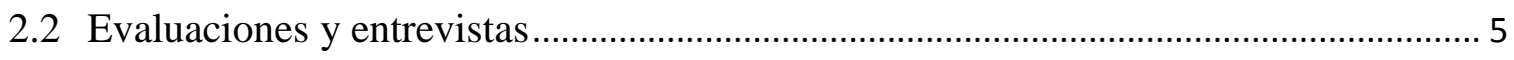

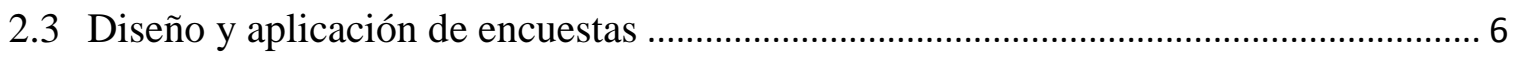

2.4 Diseño del programa de educación socio-emocional................................................... 7

CAPÍTULO III: RESULTADOS DE LA INTERVENCIÓN..................................... 10

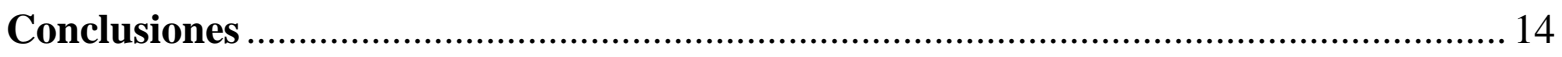

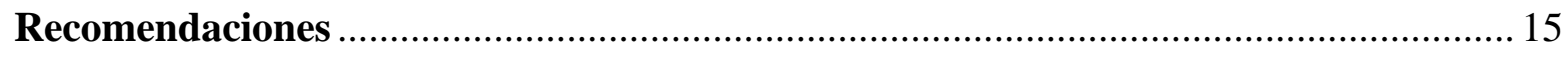

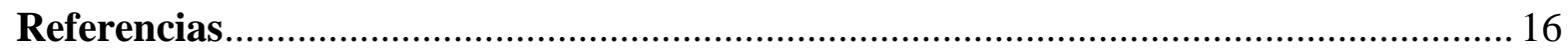

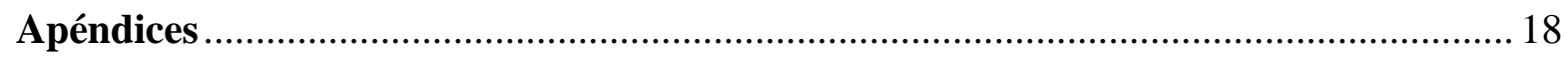




\section{ÍNDICE DE APÉNDICES}

Apéndice 1: Encuesta para 5to grado de primaria

Apéndice 2: Esquema del Programa "Educación Emocional en 6to grado de primaria"

Apéndice 3: Programa "Educación Emocional en 6to grado de primaria".....

Apéndice 4: Ejemplo de presentación en Power Point...... .34 


\section{INTRODUCCIÓN}

La educación emocional, es un "proceso educativo continuo y permanente, que busca potenciar el desarrollo emocional como complemento indispensable del desarrollo cognitivo, constituyendo ambos elementos esenciales para el desarrollo de la personalidad integral" (Bisquerra 2012, p. 90). Actualmente, en la sociedad se presenta una serie de modificaciones a nivel educativo, familiar y tecnológico, que dificultan la adaptación de la persona y pueden llegar a tener repercusiones negativas en su salud mental. Ante esto, surge la idea de llevar a cabo una promoción de la salud a través de la educación emocional desde las instituciones educativas, para que la persona desde la infancia, pueda desarrollar dicho aspecto y, por ende, cuente con más estrategias que le permitan adaptarse a un mundo lleno de cambios.

En el Perú, son pocas las instituciones educativas que se preocupan por dar una educación integral de calidad, al proponer espacios formales en el horario escolar donde se pueda desarrollar no solo el aspecto cognitivo del alumno, sino también el socio-emocional (Organización de las Naciones Unidas para la Educación, la Ciencia y la Cultura [Unesco], 2017).

En el caso de la institución educativa, en la búsqueda de cumplir con su misión, este tema era trabajado de manera superficial. El presente trabajo, tiene como fin explicar cómo al considerar importante el desarrollo de la inteligencia emocional, realizando previamente un diagnóstico del grado, se pudo diseñar un programa de educación emocional para los alumnos que pasaban a sexto grado, una promoción conformada por 134 alumnos.

Para empezar, se indagará la problemática identificada teniendo en cuenta los contextos y factores que intervienen; luego, se describirán las actividades realizadas para responder a dicho problema y finalmente, se mencionarán los resultados obtenidos durante el proceso del diseño del programa. 


\section{CAPÍTULO I: IDENTIFICACIÓN DEL PROBLEMA}

La salud mental es un tema que cada vez cobra mayor relevancia en la sociedad. En el Perú, aún es insuficiente la respuesta por parte del sistema de salud en este aspecto, pues las cifras de los ciudadanos que sufren problemas de salud mental, que en su mayoría son de origen emocional, están en aumento. Hoy en día, muchas emociones son incomprendidas y reprimidas. Como menciona Rychtenberg (2017), alrededor del 12,5\% de la población total padecen una enfermedad de esta clase. Dentro de este rango de personas atendidas, Cutipé manifiesta que el $70 \%$ de pacientes son niños y adolescentes y que los motivos por los que van se relacionan a la depresión o ansiedad (como se citó en Sausa, 2018).

En este sentido, educar a la población en relación a cómo fomentar la salud mental es de suma importancia, especialmente porque en la actualidad, tal como indica Malaisi (2016), la sociedad se encuentra inmersa en un mundo complejo y de constantes cambios, lo cual se debe en gran parte a la tecnología.

Un buen manejo de los medios digitales puede tener efectos positivos. Sin embargo, en el caso de los niños y adolescentes, su uso excesivo puede contribuir a la dependencia, depresión y ansiedad. Asimismo, puede generar dificultad para que construyan su propia identidad, dado que tienden a desarrollarla a través de las redes sociales y no necesariamente van a representar quienes realmente son (Dobrowsky, 2012).

Por otro lado, es importante mencionar que en los últimos años se han producido grandes cambios en la dinámica familiar. Los divorcios en Lima Metropolitana han aumentado en los últimos años y también, cada vez hay más madres que se dedican a trabajar (Instituto Nacional de Estadística e Informática [INEI], 2016). Estos cambios pueden llegar a desencadenar en los hijos conductas inapropiadas, problemas de ansiedad, estrés o incluso depresión, si no cuentan con las estrategias para saber afrontar dichas situaciones. 
Ante este escenario, la Organización Mundial de la Salud (OMS, 2018), señala que "las políticas nacionales de salud mental no deben ocuparse únicamente de los trastornos mentales, sino reconocer y abordar cuestiones más amplias que fomentan la salud mental como la incorporación de la promoción de la salud" (párr. 11). Es importante mencionar que, en lo que respecta a la promoción de la salud, se hace énfasis en las acciones para mejorar y mantener la calidad de vida, lo cual sería esencial para hacer frente a los cambios que se dan en la sociedad actual.

Bajo esta propuesta, para ejecutar un plan basado en la promoción de la salud, es fundamental la participación de diferentes sectores como el de educación. Ante esto, Lucas Malaisi (2016) argumenta la necesidad de una ley de educación emocional, que fomente el desarrollo de este aspecto en la persona a través de un espacio formal en la institución educativa.

En el Perú, se tiene como fin dar una educación de calidad, en la cual se desarrolle no solo el aspecto cognitivo, sino también el socioemocional del estudiante. Por ejemplo, en el Currículo Nacional de la Educación Básica, se puede encontrar que para los niveles de Inicial, Primaria y Secundaria, se brinda una orientación tutorial que tiene como finalidad un adecuado desarrollo social y afectivo del niño y adolescente, a lo largo de la etapa escolar (Ministerio de Educación del Perú [Minedu], 2017). No obstante, esta orientación no siempre es desarrollada adecuadamente y queda muchas veces en el papel, mas no en la práctica. Además, si bien existe un departamento psicopedagógico en las instituciones educativas, en la mayoría se ha hecho muy poco para educar a los estudiantes en el área socioemocional ("Educación emocional en la escuela", 2017, párr. 8).

De la misma manera, existe una problemática en la forma en que educamos en el colegio a los estudiantes, dado que se brinda una mayor importancia al aspecto cognitivo y se deja de lado el aspecto emocional (Unesco, 2015). Si bien la escuela no es el único actor que se involucra al momento de educar emocionalmente a las personas, pues gran parte de dicho desarrollo 
dependerá de la familia, el colegio puede influir a través de su llegada a todos los niños y adolescentes.

Existe una ausencia de espacios formales y estructurados en las instituciones educativas, que tracen como objetivo fomentar y desarrollar el aspecto socioemocional del alumno. Es por ese motivo que en la institución educativa, se planteó la idea de diseñar un programa para tutoría, que busque desarrollar la inteligencia emocional de los alumnos que pasarían a sexto grado. Para este, se tendría en cuenta las necesidades de los estudiantes y los beneficios que la educación emocional genera en las personas.

El colegio es una institución educativa privada y católica, que tiene como misión brindar una educación integral, formar personas íntegras, éticas y proactivas. El alumnado abarca los niveles de Inicial, Primaria y Secundaria y cada grado está conformado por un aproximado de ciento treinta estudiantes, quienes están divididos en cuatro aulas.

Es en base a la misión, que el colegio se preocupa por educar de manera integral a los alumnos. No solo le dan énfasis a lo académico, que se relaciona con el aspecto cognitivo, sino que busca el desarrollo de las habilidades socioemocionales a través de la introducción de los valores en las clases y de algunas horas de tutoría. Sin embargo, la ausencia de una programación estructurada y de un espacio formal, cuyo objetivo sea desarrollar la inteligencia emocional del estudiante, genera una falta de acción por parte de los docentes y del área psicopedagógica para trabajar este aspecto de la persona. Esto conlleva a que exista un enfoque centrado en los alumnos que ya presenten alguna dificultad y no se atiendan las necesidades que en general, el grado o los alumnos del colegio necesitan para desarrollarse de manera integral. 


\section{CAPÍTULO II: DESCRIPCIÓN DE LAS ACTIVIDADES Y TAREAS REALIZADAS}

Con el fin de abordar el problema identificado, se llevaron a cabo actividades a lo largo del año 2017. A continuación, se describirá cómo las observaciones, evaluaciones, entrevistas y encuestas, sirvieron de base para el diseño del programa.

\subsection{Observación en aula y recreo}

A lo largo del año, una de las actividades que más se realizó fue la de hacer observaciones de aula y recreo y los registros anecdóticos correspondientes, con el objetivo de observar y registrar la conducta de los estudiantes con comportamientos inapropiados o problemas emocionales detectados. En los primeros meses, las observaciones estaban enfocadas en los casos derivados al equipo psicopedagógico y los registros anecdóticos se presentaban de manera descriptiva.

A partir del mes de julio, se optó por realizar observaciones a nivel de aula con el fin de conocer la dinámica de clase. Es por ese motivo, que se realizaron entre dos a tres observaciones por aula durante el horario de clases y otras dos o tres observaciones durante la hora de recreo.

En todos los casos, la observación fue no participante, con el fin de no intervenir en la dinámica y de poder obtener mayor información de cómo son las relaciones que se establecen durante la cotidianidad. Lo observado se registró de manera narrativa y se entregó a la psicóloga del grado, con quien se tuvo reuniones para conversar sobre todo lo observado con más profundidad.

\subsection{Evaluaciones y entrevistas}


Se llevan a cabo evaluaciones psicológicas con el fin de explorar y analizar el comportamiento de la persona, tal como señala Fernández Ballesteros (1995). Entre las pruebas más utilizadas en los alumnos derivados al departamento psicopedagógico se encuentran: como pruebas proyectivas, la Evaluación con Figura Humana de Machover, el Test de Dibujo de la Familia de Corman; como prueba psicométrica, el Cuestionario de Autoconcepto de Garley. Una vez calificadas las pruebas, se redactaron informes individuales a partir de los resultados obtenidos, los cuales eran revisados por la psicóloga a cargo del grado.

Asimismo, se entrevistó al alumno, ya que la entrevista es una técnica que permite indagar a profundidad información del entrevistado y adaptarse a la necesidad de este (Poblete, Fernández, Campo y Nöel, 2014). Específicamente, en el caso de los estudiantes se buscaba recoger datos en base a sus fortalezas, su percepción en función a cómo se sentía en las diferentes áreas de su vida y cuáles eran las necesidades o dificultades que tenía. Eso permitía conocer más sobre la realidad del estudiante y complementar la información obtenida en el informe. Además, es importante mencionar que los datos obtenidos en la entrevista se redactaban en función a las áreas conversadas, para que la psicóloga del grado cuente con dicha información al momento de hacer la devolución de resultados a los padres de familia.

\subsection{Diseño y aplicación de encuestas}

Se vio necesario saber más sobre los estudiantes. En este sentido, el objetivo era conocer la realidad del grado en cuanto a distintas áreas de su vida. Por ese motivo, con la psicóloga del grado se planteó las áreas que se querían indagar: autoconocimiento, relaciones familiares, relaciones entre compañeros, tutoría, gustos e intereses y redes sociales. 
Luego, se creó la encuesta en Google Forms (Apéndice 1) por los siguientes motivos: la practicidad para crearla, la rapidez para ser llenada por todos los alumnos y para poder obtener información y organizarla de una manera más eficiente a través de Excel (Díaz de Rada, 2012). Acto seguido, se formularon preguntas que puedan brindar información con respecto a cada área y cuyo tipo de respuestas variaría, siendo un texto de respuesta corta, texto de respuesta larga o de selección múltiple. Vale recalcar que, para una mejor organización de los datos, se hicieron encuestas divididas por sección.

Para su aplicación, se coordinó con los tutores del grado destinar una hora de tutoría para que los alumnos realicen la encuesta. En la fecha pactada, los alumnos se dirigieron a la biblioteca, donde las computadoras ya se encontraban con el link de la encuesta abierto y se les explicó que debía ser llenada de manera individual. Para supervisar y aclarar dudas en el proceso, dos personas del equipo psicopedagógico, que podían ser la psicóloga del grado y una practicante o ambas practicantes, se quedaban en la biblioteca con los alumnos. Una vez que todos los estudiantes del grado llenaron la encuesta, se organizó los resultados por salón y por área y se plasmó la data obtenida en tablas y gráficos de barra.

\subsection{Diseño del programa de educación emocional}

Después de las observaciones, evaluaciones, entrevistas y especialmente, de las encuestas realizadas, se pensó en diseñar un programa que responda a las necesidades de los estudiantes y les brinde el conocimiento y las estrategias que los puedan ayudar a construir su propia identidad y educarlos emocionalmente. El plan era diseñar un programa para el año 2018, es decir, cuando ellos estén en sexto de 
primaria y que se cierre con la actividad "Encuentro de Padres e Hijos" que se lleva a cabo cuando los estudiantes están por finalizar el año.

Para esto, se realizó una reunión con la psicóloga del grado y la coordinadora encargada del área de Tutoría y Orientación Educativa, para solicitar que el siguiente año se pueda ejecutar el programa. Este tendría un enfoque sistémico en el sentido que, se buscará trabajar de manera integral con los estudiantes, el equipo psicopedagógico, los tutores y los padres de familia, pues es así que realmente se puede generar un cambio (Malaisi, 2016).

Una vez otorgado el permiso, se investigó sobre el tema y se procedió a realizar el esquema del programa (Apéndice 2), conformado por trece sesiones, en las que se trabajaría el autoconocimiento, la autoestima, las relaciones con los demás, las emociones y la familia. Se propuso dividir al salón en dos grupos, para que el taller se lleve a cabo de manera más personalizada y se cuente con mayor espacio. Cada grupo conformado por dieciséis o diecisiete alumnos, estaría dirigido tanto por el tutor como por la psicóloga del grado, lo que generaría que se lleve a cabo un trabajo integrado y que haya un acompañamiento directo a los tutores. Cabe recalcar, que los docentes propusieron agrupar las sesiones por temas. Por ende, el esquema final se basó en seis temas a trabajar, cada uno con una respectiva cantidad de sesiones. En el diseño del programa, se dedicaron dos sesiones para hacer una introducción e integrar a los grupos establecidos. La tercera y cuarta, se dedicaron al tema del autoconocimiento (Apéndice 3); la quinta y sexta sesión, se relacionaron al tema de la autoestima; la sétima y octava sesión, fueron en función a la relación con los demás; la novena, décima, décimo primera y décimo segunda, bajo el tema de las emociones; por último, la décimo tercera sesión se diseñó bajo el tema de familia, pues sería la anterior al "Encuentro de Padres e Hijos". 
Una vez que el esquema fue aprobado, se redactó una circular dirigida a los padres de familia, para que estén al tanto del programa que se planeaba desarrollar con sus hijos y puedan acompañarlos en todo este proceso, pues la familia tiene un rol fundamental en este aspecto (Trinidad y Latorre, 2012).

Luego, se empezó con el desarrollo de cada sesión y se consideró que el programa debía tener una metodología lúdica, participativa y reflexiva, pues lo que se quiere es que los alumnos sean los protagonistas de dicho programa. Por otro lado, para el planteamiento de cada sesión se tuvo en cuenta: el objetivo de cada una, las actividades que se podían llevar a cabo para desarrollar el tema propuesto que logren motivar y captar el interés de los alumnos, los materiales a utilizar y finalmente, ideas claves para que la persona que vaya a guiar la sesión tenga claro cuál es el mensaje que se busca transmitir.

Una vez redactadas, se confeccionó el material anexo necesario para cada sesión, como las tarjetas, imágenes, búsqueda de videos, entre otros. Además, se elaboró una presentación en Power Point (Apéndice 4) para acompañar las sesiones con un recurso visual.

Por último, se pensó en la manera de poder evaluar el programa al ser finalizado. Para ello, se formuló una serie de indicadores que permitan medir de forma objetiva, el impacto del programa en función a los alumnos, tutores, la psicóloga del grado y los padres de familia. Asimismo, para complementar dicha información, se propone que los tutores dialoguen con sus alumnos y que la psicóloga del grado se reúna con los tutores, para pedir una retroalimentación del programa y poder conocer qué aspectos positivos y negativos tuvo, con el fin de optimizarlo para una futura aplicación. 


\section{CAPÍTULO III: RESULTADOS DE LA INTERVENCIÓN}

En términos generales, se puede decir que el proceso previo al diseño del programa para los estudiantes que pasarían a sexto grado de primaria fue de mucha utilidad. Las observaciones, evaluaciones, entrevistas y encuestas permitieron tener una idea de las necesidades del grado, de lo que les llamaba la atención y les interesaba. Esto permitió llevar a cabo un programa que integre dichas necesidades e intereses con el educar emocionalmente a los alumnos, pues tal como sugiere Bisquerra (2012), se tiene que realizar un análisis de las necesidades que tengan los estudiantes para a partir de ahí, establecer objetivos a trabajar y plantear el contenido que se pondrá en práctica a través de actividades.

Al inicio, se trabajó solo con los alumnos que eran derivados al departamento psicopedagógico. En total, fueron trece alumnos observados, de los cuales a cuatro se les vio por conductas inapropiadas, a cuatro por problemas a nivel emocional, a dos por problemas familiares, a dos por problemas de aprendizaje y a dos por dificultades en la socialización. En cuanto a las evaluaciones y entrevistas, se realizaron a tres de los alumnos con problemas emocionales, a uno por problemas familiares, uno por conductas inapropiadas y uno por dificultades en la socialización.

En general, se encontró que de los seis alumnos que habían pasado por los tres procesos, en cuanto a su autoconocimiento, el $83 \%$ tenían dificultades en cuanto a identificar sus fortalezas, aspectos a mejorar y características; en relación a las relaciones interpersonales, el 67\% sentían que les costaba establecer relaciones significativas con los demás y en función a su dinámica familiar, el 50\% sentían que debía mejorar.

Por otro lado, a nivel de grado, fueron las observaciones realizadas sobre la dinámica de clase y recreo y las encuestas, lo que brindó más información. En cuanto a las observaciones, se pudo encontrar lo siguiente: algunos alumnos decían comentarios agresivos o burlas y con ello herían 
a los demás, solo dos estudiantes llegaron a tener una conducta física agresiva, la cuarta parte del salón aproximadamente se aburría en clase y les costaba atender al docente. En cuanto a la clase de tutoría, muchos no estaban interesados en el tema y pedían al tutor hacer una actividad lúdica. Asimismo, se detectó a un grupo de nueve niños que se consideraban los populares de la promoción y que buscan el reconocimiento y la atención de los demás, lo que generó que otros compañeros quieran pertenecer a su grupo. También, se observaron estudiantes que paraban solos en el patio, que preferían ir a la biblioteca y que durante las clases tenían pocas interacciones con los demás.

Con respecto a la encuesta, se alcanzó un $100 \%$ de participación de los alumnos de quinto grado, lo cual permitió obtener información no solo de cada uno, sino también del grado en su totalidad. Entre los resultados obtenidos más relevantes, en cuanto al área escolar, se halló que $76 \%$ de la promoción estaba interesada en que el siguiente año durante la clase de tutoría, se profundice en temas sobre las emociones, autoconcepto, autoestima y amistad. Por otro lado, en relación a las dinámicas de trabajo en clase, el $63 \%$ prefería que sean a través de trabajos de exploración y trabajos creativos.

Según los datos en base al autoconocimiento, al $68 \%$ de los alumnos les costó identificar sus fortalezas, dificultades y características propias. En relación al área de relaciones interpersonales, se halló que lo que más aprecian en una amistad, son los valores que el otro tenga, especialmente la amabilidad, el respeto y la honestidad. Asimismo, se pudo identificar según la percepción de cada uno, quiénes eran los alumnos que molestaban a los demás, cómo lo hacían, quiénes eran a los que solían molestar y quiénes paraban solos en los recreos. Toda esta información fue útil para hacer un seguimiento y elaborar un plan de acción. Por último, en el área de los intereses, se halló que el 100\% de alumnos contaban con al menos, una red social. 
Los resultados obtenidos fueron muy interesantes y se tomaron en cuenta para elaborar el programa de educación emocional. Esta herramienta fue de mucha utilidad y quedó como un aporte para la institución, ya que era la primera vez que se realizaba una encuesta de manera digital, con preguntas basadas en aspectos de estrecha relación al alumno. Además, permitía tener información sobre cada uno de ellos y a nivel de grado, el cual era el objetivo de esta actividad. Al mismo tiempo, permitió organizar los resultados de manera más eficiente, lo cual fue significativo no solo para la psicóloga del grado, sino también para los tutores, docentes y coordinadores. Fue a partir de esta encuesta y el impacto positivo que tuvo, que en los meses siguientes se adaptó el formulario para aplicarlo en tercer grado de primaria.

Toda la información obtenida, se tomó en cuenta a la hora de diseñar el programa de educación emocional. Al inicio, hubo cierta resistencia por parte de los docentes, ya que al comunicarles que se deseaba ejecutar un programa en la hora de tutoría basado en la inteligencia emocional, se preocuparon por el tiempo que esto implicaba y por la sobrecarga laboral. No obstante, al explicarles que sería elaborado por el departamento psicopedagógico y que se basaría no solo en la teoría y etapa evolutiva del alumno, sino también en la información recogida previamente, cambiaron su actitud y estuvieron de acuerdo. Al entregar el esquema terminado al área psicopedagógica, coordinación de Tutoría y Orientación Educativa y tutores del grado, este tuvo gran aceptación y un impacto positivo, pues se apreció que efectivamente, es importante educar en este aspecto al estudiante y les gustaría ver el resultado.

Debido a que solo se contaba con trece sesiones para desarrollar el programa, se abarcó todos los temas propuestos, dándole una mayor cantidad de sesiones a los temas percibidos como más importantes y necesarios. Es por eso, que una vez trabajado el autoconocimiento y autoestima, lo cual se considera como base, se enfatizó el tema de emociones.

Cabe recalcar que, si bien se dedicaron cuatro sesiones al tema de emociones, aún hay mucho por enseñar y trabajar. Asimismo, en función a las relaciones interpersonales se buscó 
desarrollar la empatía, pues se percibió como una de las habilidades más necesarias a desarrollar en el grupo. Sin embargo, aún es necesario ahondar en este tema y en otras habilidades sociales que les ayuden a interactuar apropiadamente, como la comunicación asertiva o la negociación.

La meta planteada, fue diseñar un programa para la tutoría sobre educación emocional, que esté compuesto por trece sesiones y tenga como finalidad el desarrollar la inteligencia emocional de los alumnos de sexto grado de primaria, en un plazo de dos meses. Por ese motivo, se puede decir que al cumplirla, se generó un gran aporte y tuvo un impacto positivo en la institución al ser tomado con entusiasmo por la coordinación de las distintas áreas y con una buena disposición a llevarlo a cabo por parte de los tutores. Además, la coordinación opinó que, con este programa, se empezaría a abordar la problemática de la institución y que se podría tomar como base de futuros programas que puedan ser aplicados a lo largo de la etapa escolar. 


\section{CONCLUSIONES}

- La educación emocional es un proceso educativo que permite el crecimiento integral de la persona. Por ese motivo, es importante que pueda desarrollarse en la institución educativa a través de espacios formales y del trabajo colaborativo entre distintas áreas.

- Las observaciones, evaluaciones, entrevistas y encuestas, fueron de gran utilidad para hacer un diagnóstico general del grado y conocer cuál era una necesidad a trabajar en relación al aspecto psicológico de los alumnos.

- El tener conocimientos previos sobre el grupo con el que se trabajará, permite contar con más recursos en el sentido de involucrar sus necesidades, gustos e intereses en el plan para abordar el problema identificado, desde una perspectiva que los involucre y motive a que sean partícipes del proceso.

- Con respecto al diseño del programa, es importante que se pueda investigar sobre el tema a desarrollar, así como pensar en actividades que pongan al alumno como protagonista, tomar en cuenta a la familia e institución educativa y plantear indicadores que permitan conocer los resultados una vez que se aplique el programa.

- El contar con un programa ya hecho por el equipo psicopedagógico y el tener la autorización de llevarlo a cabo durante las clases de tutoría del siguiente año, permite que se pueda abordar a profundidad el desarrollo socioemocional del alumno, cumpliendo así con la misión del colegio: brindar una educación integral de calidad. 


\section{RECOMENDACIONES}

- Implementar a nivel institucional un programa para cada grado, que tenga como objetivo el desarrollo de la inteligencia emocional en los estudiantes, tomando en cuenta las necesidades del grupo.

- Aplicar la técnica del focus group para indagar sobre las necesidades e intereses de los alumnos, lo cual permitiría escuchar sus opiniones en menos tiempo y generaría mayor conocimiento sobre el grado.

- Llevar a cabo capacitaciones o talleres que permitan una formación a nivel institucional sobre la inteligencia emocional, para el beneficio propio y de los estudiantes, pues todos son agentes activos en la cotidianidad en cuanto a este aspecto.

- Que el área psicopedagógica desarrolle actividades que abarquen una promoción o prevención de la salud y no centrarse en qué hacer cuando el problema ya apareció.

- Se recomienda aplicar el programa en los alumnos de sexto grado y que, una vez finalizado, se analicen los resultados para que a partir de estos, se hagan las modificaciones necesarias con la intención de mejorarlo.

- Para poder analizar el impacto que tiene el desarrollo de la inteligencia emocional en el alumno a través del programa, este debe tener una continuidad y seguimiento. Por lo tanto, se debe seguir desarrollando este aspecto una vez que pasen a secundaria. 


\section{REFERENCIAS}

Bisquerra Alzina, R. (2012). Orientación, tutoría y educación emocional. Madrid: Síntesis.

Díaz de Rada, V. (2012). Ventajas e inconvenientes de la encuesta por internet. Papers, 97(1), 193-223. Recuperado de https://papers.uab.cat/article/view/v97-n1-diaz/pdf

Dobrowsky, D. (2012). Constructing identity on social networks. An analysis of competences of communication constituted on Facebook.com. Central European Journal of $\begin{array}{llll}\text { communication. } & 5(1), & \text { 91-103. } & \text { Recuperado }\end{array}$ http://cejsh.icm.edu.pl/cejsh/element/bwmeta1.element.desklight-c7b8d2ab-38b24078-a3d1-6b5a062f54d7

Educación emocional en la escuela. (13 de marzo de 2017). Recuperado de http://proexpansion.com/es/articulos_oe/2884-educacion-emocional-en-la-escuela

Fernández Ballesteros, R. (1995). Introducción a la evaluación psicológica. Madrid: Pirámide.

Instituto Nacional de Estadística e Informática. (2016). Natalidad, Mortalidad y Nupcialidad, 2016. Recuperado

de https://www.inei.gob.pe/media/MenuRecursivo/publicaciones_digitales/Est/Lib1485/1 ibro.pdf

Malaisi, L. (2016). Cómo ayudar a los niños de hoy: educación emocional. San Juan: Educación Emocional Argentina.

Ministerio de Educación. (2017). Programa curricular de educación primaria. Recuperado de http://www.minedu.gob.pe/curriculo/pdf/programa-primaria-16-marzo.pdf

Organización de las Naciones Unidas para la Educación, la Ciencia y la Cultura. (2015). Replantear la educación. ¿Hacia un bien común mundial?. Recuperado de http://unesdoc.unesco.org/images/0023/002326/232697s.pdf 
Organización de las Naciones Unidas para la Educación, la Ciencia y la Cultura. (2017). Revisión de las políticas públicas del sector de educación en Perú. Recuperado de https://unesdoc.unesco.org/ark:/48223/pf0000260875

Organización Mundial de la Salud. (30 de marzo de 2018). Salud mental: fortalecer nuestra respuesta. Recuperado de https://www.who.int/es/news-room/factsheets/detail/mental-health-strengthening-our-response

Poblete, M., Fernández, D., Campo, L. y Nöel, M. (2016). La entrevista como herramienta para la evaluación de competencias genéricas: construcción de un consenso sobre evidencias. Revista Complutense de Educación, 27(2), 457-476. http://dx.doi.org/10.5209/rev_RCED.2016.v27.n2.46375

Rychtenberg, C. (2 de febrero del 2017). Salud mental en el Perú. Recuperado de http://noticias.universia.edu.pe/cultura/noticia/2017/02/01/1149001/salud-mentalperu.html

Sausa, M. (03 de febrero de 2017). Menores ocupan el 70\% de atenciones en salud mental [INFOGRAFÍA]. Perú 21. Recuperado de https://peru21.pe/peru/cifras-salud-mentalperu-menores-ocupan-70-atenciones-infografia-394376

Trinidad, M. y Latorre, J. (2012). Inteligencia emocional y clima familiar. Psicología $\begin{array}{llll}\text { Conductual, } & \text { 20(1), } & \text { 103-117. } & \text { Recuperado de }\end{array}$ http://go.galegroup.com.ezproxy.ulima.edu.pe/ps/i.do?p=IFME\&u=ulima\&id=GALE| A314254292\&v=2.1\&it=r\&sid=summon\&authCount $=1$ 
APÉNDICES 


\section{APÉNDICE 1: ENCUESTA PARA QUINTO GRADO}

\section{Encuesta 5to grado A}

Responde las sigulentes preguntas que nos servirán para conocer un poco más de ti, lo que te gusta hacer, tus intereses y cómo te estás sintiendo este año en el colegio. Marca en los espacios sefialedos.

*Obligatorio

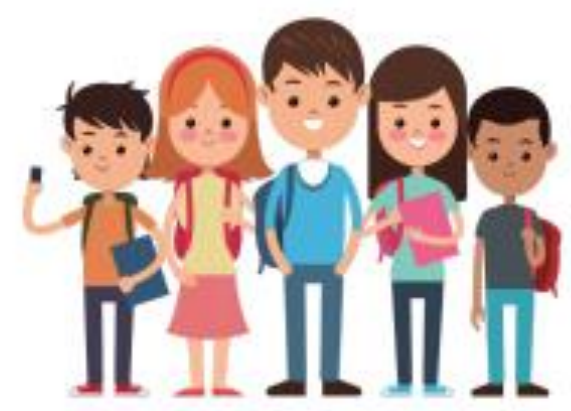

1. Nombre y apellido: *

\section{Sexo: *}

Marca solo un dvalo.

Masculino

Femenino

3. Edad: "

Marca solo un dvalo.

10 antos

11 años

12 antos

4. ¿Con quién vives en tu casa? *

Selecciona todos los que correspondan.
Mama
Papa
Hernamols(a/as)
Otro: 
5. ¿Con quién de tu familia conversas más y le confias tus problemas? *

6. ¿Cómo te describes tu forma de ser? *

7. Menciona 2 cualidades fisicas y 2 emocionales con las que más te identifiques: *

8. Dentro de tus caracteristicas, ¿cuáles son tus fortalezas y/o habilidades? "

9. ¿Qué aspectos crees que necesitas mejorar como persona? *

\section{Relación con compañeros}




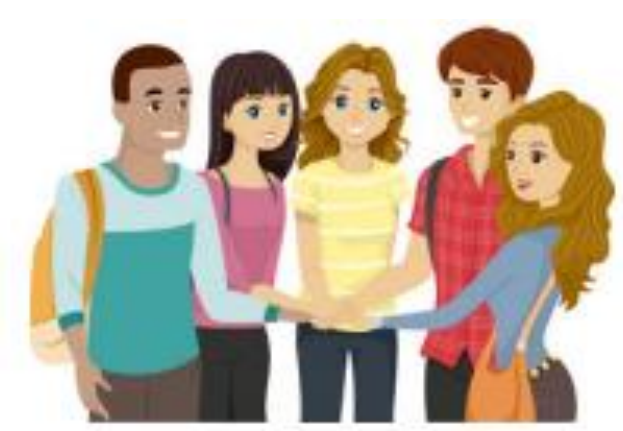

10. ¿Cómo te sientes con tus compañeros de aula? * Marca solo un ovalo.
Muy bien
Bien
Regular
Mal
Muy ma

11. Menciona 1 o 2 compafieros(as) de tu salón con quien te sientes más cercano y a gusto. (Coloca su nombre y apellido) *

12. Menciona a 2 o 3 compafteros(as) de otras secciones de tu grado que son parte de tu grupo de amigos(as). *

13. Menciona 1 o 2 companeros(as) de tu salón que suelen molestar mucho. (Coloca su nombre $y$ apellido) *

14. En base a la pregunta anterior, ¿qué hacen estos compañeros para molestar a los demás? * 
15. Menciona 1 o 2 compafieros(as) de tu salón que es fastidiado por otros nifios(as) (Coloca su nombre y apellido) ?

16. Menciona 1 o 2 compaferos(as) de tu salón a quienes sueles ver solos(as) en el recreo u otros momentos libres. (Coloca nombre y apellido) *

\section{Amistad}

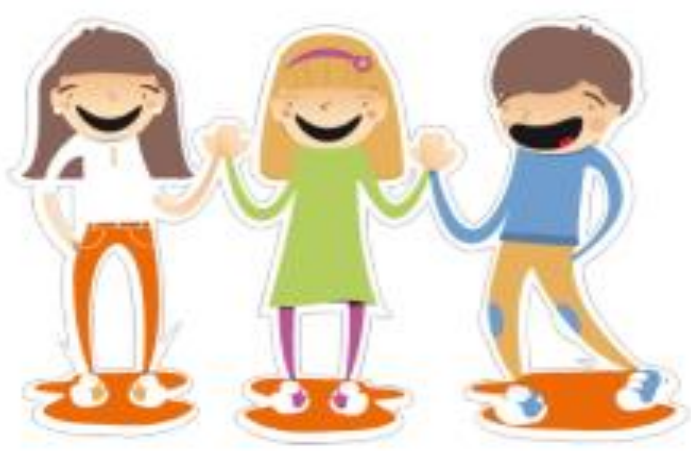

17. ¿Qué es lo que más valoras en un amigo(a)? * 
18. ¿Qué es lo que más te atrae o interesa para que formes parte de un grupo de amigos(as)? (marcar hasta 3 opciones) *

Selecciona todos los que correspondan.

Que sean creativos, ingeniosos, innovadores

7 Que sean buenos estudiantes

Que sean divertidos

7 Que sean populares

1 Que practiquen el deporte que me gusta

1 Que se muestren sinceros y de conflanza

Que sean buenas personas y con buenos sentimientos

Otro:

19. ¿Qué cualidades son las que te pueden llegar a gustar en un compafiero(a) del sexo opuesto?

20. ¿Qué harias tú por un amigo que se encuentra en un momento dificil? *

21. ¿Cómo te gustaria que te apoyen tus amigos si te encontraras en una situación dificil? *

\section{Colegio}




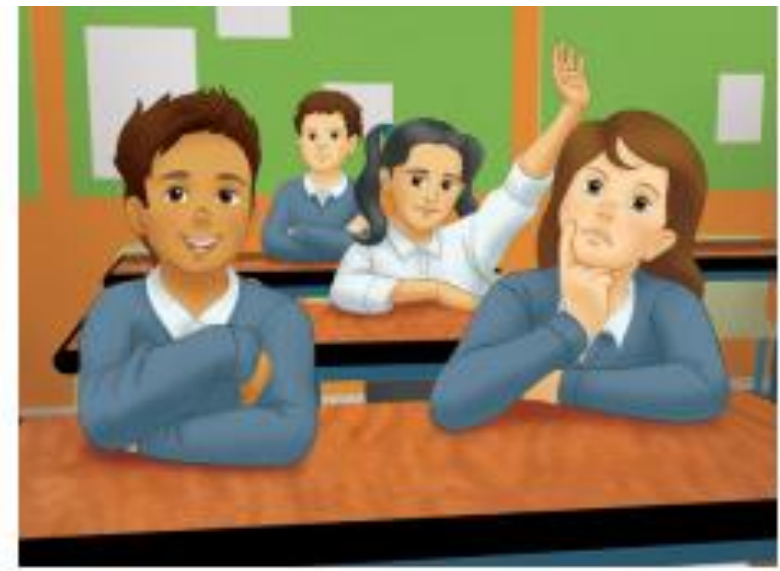

22. ¿Qué temas nuevos te gustaria que se trabajen en Tutoria? (Ejemplos: Hablar sobre el enamoramiento, una amistad verdadera, autoestima, etc.) Menciona 4 que sean de tu preferencia e interés. *

23. ¿Cómo te gustaria que sean las dinámicas de trabajo en clase? (se puede marcar más de 1 opción) *

Selecciona todos los que correspondan.

Trabajo de exploración como visitas guiadas de estudios

Debates sobre temas

7. Trabajos audiovisuales (power point, escuchar audios, computadoras, etc)

] Trabajos creativos (cuentos, manualidades, maquetas, etc)

Dinámicas motivadoras en clases (ludicas/)uegos)

7 Trabajo de investigacion (en diversos temas)

T Trabajos de resúmenes, mapas conceptuales, esquemas

Otro:

24. ¿Qué norma de convivencia nueva propondrias para el aula? " 
25. En un trabajo grupal, ¿cuál es el rol con el que puedes colaborar y sentirte bien? * Marca solo un ovalo.

Coordinador: Es el que lidera al equipo para que todos realicen las distintas tareas de manera adecuada.

Innovador: Le gusta brindar ideas creativas y es bueno resolviendo problemas.

Irvestigador: Busca informacion y proporciona conocimiento.

Motivador: Ayuda a que el equipo se sienta mas unido y que trabajen todos juntos animándolos.

Finalizador: Observa los citimos detalles del trabajo antes de entregarlo, decora, corrige errores, etc.

\section{Intereses}

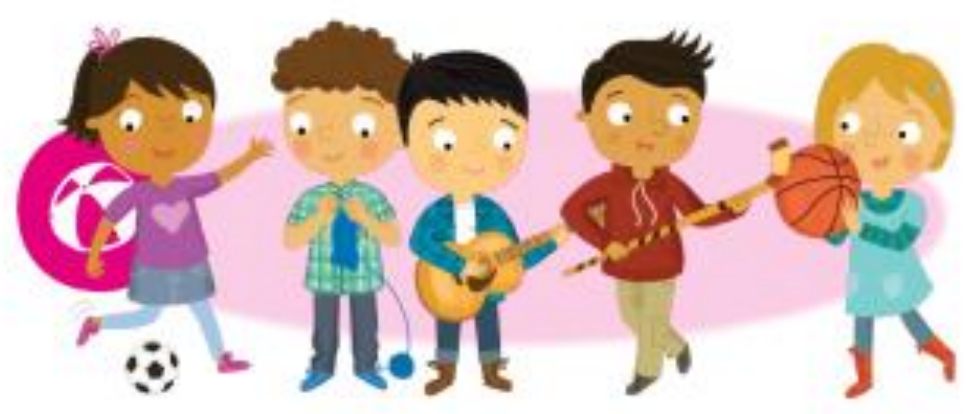

26. ¿Cuáles son las cosas que te gustan hacer? "

27. ¿Qué juegas en el recreo? *

28. ¿Que sueles jugar en casa o con amigos? (Escribe por lo menos tres juegos) * 
29. ¿Cuáles son tus cantantes o grupos de música favoritos? *

30. ¿Qué tipo de peliculas te gusta ver? Puedes marcar 2 respuestas * Selecciona todos los que correspondan.

$\square$ Acción

$\square$ Comedia

$\square$ Ciencia-ficción

7nimadas

$\square$ Terror

$\square$ Drama

31. ¿Qué redes sociales utilizas más seguido? Puedes marcar 2 respuestas " Selecciona todos los que correspondan.

1] Facebook

Instagram

Whatsapp

Youtube

Snapchat

$\square$ Otro:

Con la tecnologia de

由 Google Forms 


\section{APÉNDICE 2: ESQUEMA DEL PROGRAMA DE EDUCACIÓN EMOCIONAL}

Con el permiso de la institución educativa, se permitió presentar el esquema del programa diseñado.

\section{$\underline{\text { Programa de Educación Emocional - 6to grado }}$}

\section{Estructura del Programa}

\section{Fundamentación teórica}

La inteligencia emocional es un concepto utilizado a nivel mundial, al cual se le ha ido otorgando una mayor importancia en los últimos años. Mayer y Salovey (1997) la definen a partir de cuatro habilidades:

- La habilidad para percibir, valorar y expresar emociones con exactitud.

- La habilidad para acceder y/o generar sentimientos que faciliten el pensamiento.

- La habilidad para comprender emociones y el conocimiento emocional.

- La habilidad para regular las emociones promoviendo un crecimiento emocional e intelectual.

Por otro lado, existe un concepto acuñado por Bisquerra (2000), el cual se origina al plantear que la inteligencia emocional es un constructo capaz de ser modificado, este término es el de educación emocional. Se le puede definir como un "proceso educativo continuo y permanente, que busca potenciar el desarrollo emocional como complemento indispensable del desarrollo cognitivo, constituyendo ambos elementos esenciales para el desarrollo de la personalidad integral" (Bisquerra, 2012, p. 90).

Investigaciones han dado como resultado que, aquellas personas que logran desarrollar su inteligencia emocional desde niños, se desenvuelven mejor en la escuela, tienen mejores relaciones interpersonales y logran un comportamiento más saludable (Rubenstein, 2017; Oros, Manucci, Richaud-de Minzi, 2011).

Asimismo, en cuanto al área académica, se conoce que los eventos que tienen una carga emocional son almacenados en la memoria con mayor intensidad. Esto quiere decir que, si bien las emociones positivas tienen un impacto a favor de la persona, como brindar mayor motivación, interés, compromiso, perseverancia, flexibilidad, entre otros; las emociones negativas tienen un efecto contrario, causando mayor estrés y dificultades emocionales en sus estudios, bloqueo, resistencia, inadecuada comunicación, ausentismo, entre otros. Esto se debe a que impactan sobre las funciones cognitivas como la percepción, el juicio, la atención, la memoria de trabajo y el razonamiento y con ello, en el rendimiento académico del estudiante (D. Chabot y M. Chabot, 2009). 
Con respecto a la institución educativa, ésta también puede aportar mucho para el desarrollo de la inteligencia emocional de sus estudiantes. Para empezar, las emociones positivas pueden irse desarrollando mediante prácticas y juegos con los compañeros de clase, que promuevan la capacidad de reconocer las propias emociones del niño y el saber cómo autorregularlas, debido a que, como menciona Bisquerra (2012), sobre ellas se basa el bienestar subjetivo de la persona.

Una manera de desarrollar la inteligencia emocional en los estudiantes, es a través de programas sobre educación emocional. Por ejemplo, Renom (2008), realiza programas específicos dependiendo de las edades que tengan los estudiantes y los diseña en base a estos cinco aspectos: conciencia emocional, regulación emocional, autoestima, habilidades socio-emocionales y habilidades de vida.

Asimismo, Trinidad y Latorre (2012), mencionan que el rol que tiene la familia en el desarrollo de la inteligencia emocional de una persona es fundamental, pues se ha considerado a la familia como "el factor de mayor fuerza en la socialización de emociones", pues es en este ambiente en el que las competencias emocionales se van formando.

\section{Justificación}

A partir de las observaciones, evaluaciones, entrevistas y encuestas realizadas, se plantea la necesidad que tiene el grado de poder desarrollar su aspecto socio-emocional. No solo con el fin de que cada uno de ellos desarrolle un autoconocimiento y autoestima, sino también para que empiecen a contar con estrategias que les permitan identificar y regular sus emociones, mejorando así sus relaciones interpersonales, siendo más empáticos con sus compañeros.

Tal como señalan Extremera y Fernández-Berrocal (2004): “Las escuelas no pueden ser indiferentes ante la necesidad de los estudiantes, es importante brindarles las herramientas emocionales necesarias para que no se vean afectados ante los riesgos que se presentan en la adolescencia" (p. 4). Con ello, enfatiza la idea de que los alumnos deben ser "alfabetizados emocionalmente" a través de talleres o programas.

Es por eso, que se considera esencial diseñar un programa de educación emocional para los alumnos de quinto grado de primaria, el cual podría ser aplicado en el horario de tutoría el próximo año. Además, este programa podría tener un cierre en la actividad "Encuentro de Padres e Hijos", en el cual se hablará de la importancia del desarrollo socioemocional de la persona.

\section{Objetivos}

\section{- General:}

- Desarrollar la inteligencia emocional de los alumnos de 6to grado, a través del autoconocimiento, autoestima, empatía y la capacidad para identificar, expresar y regular sus emociones, permitiéndoles enfrentar el día a día y poder relacionarse con las personas de su entorno de una manera más apropiada.

\section{- Específicos:}


- Construir su identidad en base a una alta autoestima, al poder identificar sus gustos, intereses, valores, características de la personalidad, fortalezas y oportunidades de mejora.

- Desarrollar su comprensión de los demás, aceptando que cada persona es diferente y que no siempre van a pensar, sentir o actuar de la manera en la que uno desea.

- Identificar, expresar y regular sus propias emociones.

- Comprender que cada familia es única y diferente y se planteen una manera de mejorar su dinámica familiar.

4. Cronograma

\begin{tabular}{|c|l|c|c|c|c|c|c|c|c|c|c|c|c|c|c|}
\hline \multirow{2}{*}{$\mathbf{N}^{\circ}$} & \multicolumn{10}{|c|}{ TEMA } & \multicolumn{9}{|c|}{ MES } \\
\hline & & 05 & 06 & 06 & 06 & 07 & 08 & 09 & 09 & 09 & 10 & 10 & 11 & 11 \\
\hline I & Bienvenida & & & & & & & & & & & & & \\
\hline II & Bienvenida & & & & & & & & & & & & & \\
\hline III & Autoconocimiento & & & & & & & & & & & & & \\
\hline IV & Autoconocimiento & & & & & & & & & & & & & \\
\hline V & Autoestima & & & & & & & & & & & & & \\
\hline VI & Autoestima & & & & & & & & & & & & & \\
\hline VII & Relación con el otro & & & & & & & & & & & & & \\
\hline VIII & Relación con el otro & & & & & & & & & & & & & \\
\hline IX & Emociones & & & & & & & & & & & & & \\
\hline X & Emociones & & & & & & & & & & & & & \\
\hline XI & Emociones & & & & & & & & & & & & & \\
\hline XII & Emociones & & & & & & & & & & & & & \\
\hline XIII & Familia & & & & & & & & & & & & \\
\hline
\end{tabular}

\section{Metodología}

A lo largo del programa de intervención, se llevarán a cabo sesiones en las cuales se utilizarán las siguientes técnicas:

- Teoría expositiva - Role Playing - Reflexión

- Casos - Fichas de trabajo - Dinámicas

- Exposiciones - Medios audiovisuales

\section{Recursos}


Los recursos que se utilizarán para este programa son:

\begin{tabular}{|c|c|c|}
\hline Pizarra & - Hojas de colores & - Tijeras \\
\hline Plumones gruesos & - Colores & - Papelógrafos \\
\hline Computadora & - Imágenes a color & Fichas de trabajo \\
\hline Goma & - Videos & Película \\
\hline Cartulina & - Tarjetas & \\
\hline
\end{tabular}

\section{Sesiones}

- Tema: Bienvenida

- Sesión I: "Empezando juntos"

- Sesión II: "Empezando juntos"

- Tema: Autoconocimiento

- Sesión III: "Me voy conociendo - Parte 1"

- Sesión IV: "Me voy conociendo - Parte 2"

- Tema: Autoestima

- Sesión V: "Me conozco, me acepto y me quiero - Parte 1"

- Sesión VI: "Me conozco, me acepto y me quiero - Parte 2"

- Tema: Relaciones interpersonales (empatía)

- Sesión VII: "Aprendiendo de nuestras diferencias - Parte 1"

- Sesión VIII: "Aprendiendo de nuestras diferencias - Parte 2"

- Tema: Emociones

- Sesión IX: "Reflexiono e identifico emociones - Parte 1"

- Sesión X: "Reflexiono e identifico emociones - Parte 2"

- Sesión XI: "Conozco y aprendo a manejar mis emociones - Parte 1"

- Sesión XII: "Conozco y aprendo a manejar mis emociones - Parte 2"

- Tema: La familia

- Sesión XIII: "Reflexionando sobre mi familia"

\section{Referencias:}

Bisquerra Alzina, R. (2000). Educación emocional y bienestar. Barcelona: Praxis.

Bisquerra Alzina, R. (2012). Orientación, tutoría y educación emocional. Madrid: Síntesis.

Chabot, D. y Chabot, M. (2009). Pedagogía emocional. Sentir para aprender. Integración de la inteligencia emocional en el aprendizaje. México D. F.: Alfaomega.

Fernández-Berrocal, P. y Extremera, N. (2005). La inteligencia emocional y la educación de las emociones desde el modelo de Mayer y Salovey. Revista Interuniversitaria de Formación del Profesorado, 19(3), 63-93. Recuperado de http://emotional.intelligence.uma.es/documentos/pdf61modelo_de_mayer_salovey.pdf 
Mayer, J. \& Salovey, P. (1997). Emotional Development and Emotional Intelligence:

Educational Implications. Recuperado de http://ei.yale.edu/publication/emotionalintelligence-4/

Oros, L. B., Manucci, V. y Richaud-de Minzi, M. (2011). Desarrollo de emociones positivas en la niñez: lineamientos para la intervención escolar. Educ. Educ. 14(3), 493-509. Recuperado de http://repositorio.minedu.gob.pe/bitstream/handle/123456789/2188/2011_Oros_Desar rollo\%20de\%20emociones\%20positivas\%20en\%20la\%20ni\%C3\%B1ez$\% 20$ Lineamientos\%20para\%201a\%20intervenci\%C3\%B3n\%20escolar.pdf?sequence= $1 \&$ isAllowed=y

Renom Plana, A. (2008). Educación emocional: programa para educación primaria de 6-12 años. (3. ${ }^{\mathrm{a}}$ ed.). Madrid: Wolters Kluwer.

Rubenstein, G. (24 de febrero del 2017). Should emotions be taught in schools? [mensaje en un blog]. Recuperado de http://blog.ed.ted.com/2017/02/24/should-emotions-betaught-in-schools/

Trinidad, M. y Latorre, J. (2012). Inteligencia emocional y clima familiar. Psicología Conductual, 20(1), 103-117. Recuperado de http://go.galegroup.com.ezproxy.ulima.edu.pe/ps/i.do?p=IFME\&u=ulima\&id=GALE A $314254292 \& v=2.1 \& i t=r \&$ sid $=$ summon\&authCount $=1$ 


\section{APÉNDICE 3: PROGRAMA DE EDUCACIÓN EMOCIONAL}

Con el permiso de la institución educativa, se permitió presentar una sesión del programa.

PROGRAMA DE “EDUCACIÓN EMOCIONAL”

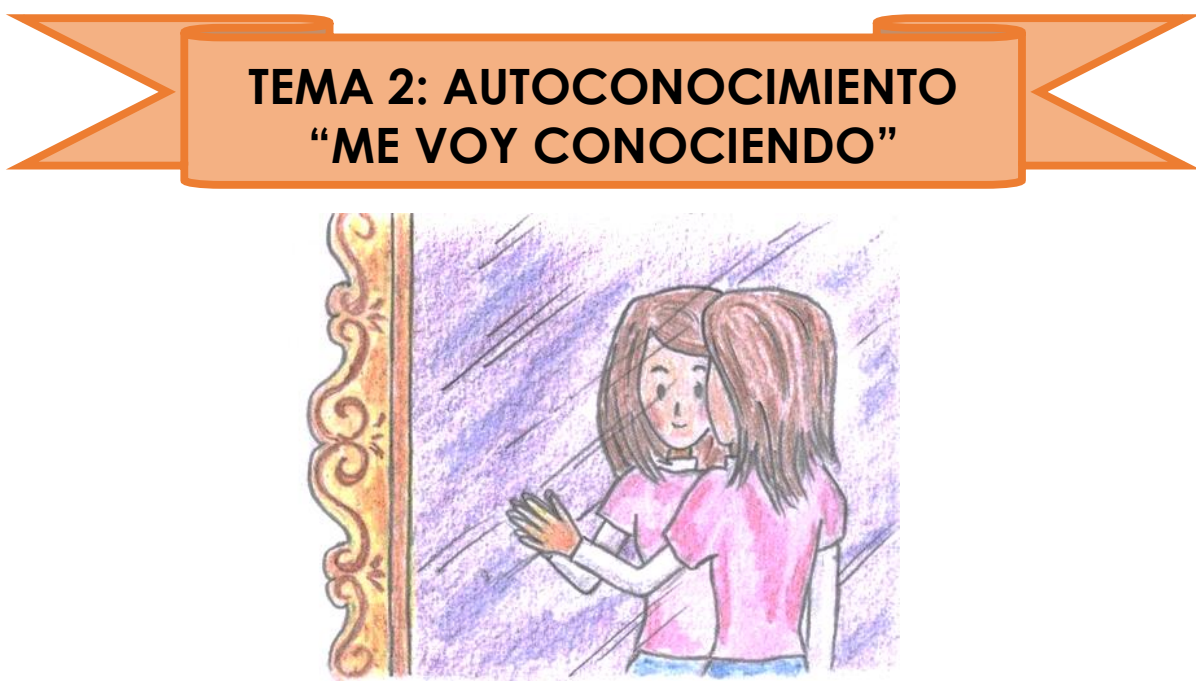

\section{OBJETIVO}

Que el estudiante pueda identificar sus habilidades, fortalezas, características, valores y aspectos a mejorar, con el fin de conocerse a sí mismo e ir construyendo su identidad.

\section{PROCEDIMIENTO}

\section{Sesión III}

- Bienvenida a los alumnos con mucho entusiasmo. Se les lee rápidamente lo que se realizará a lo largo de la sesión de hoy. Ver agenda en ppt (3 min).

- Dinámica de Bingo: Para empezar, se les entrega las hojas de Bingo vacías y se les explica la actividad: "Se mostrará en la diapositiva (ppt) un cuadro con distintas palabras que representen cualidades y de las cuales cada uno deberá escoger 3 fortalezas y 3 oportunidades de mejora con las que se identifiquen más. Las escribirán en el cuadro de Bingo según corresponda". Una vez que todos hayan escrito las palabras en su cartilla, la profesora irá sacando al azar de la bolsa, cada una de las palabras (de manera intercalada, es decir una fortaleza y una oportunidad de mejora) y el alumno marcará con una $X$ la palabra que coincida. El primero que complete su tablilla debe gritar "¡Bingo!" y tendrá un premio sorpresa (15 min).

- Preguntas para reflexionar:

1. ¿ंFue más fácil identificar las fortalezas que las cualidades a mejorar? ¿Por qué?

2. ¿ ¿Cómo uno reconoce las fortalezas que tiene?

3. ¿̇En qué situaciones pensamos más en los aspectos a mejorar?

4. ¿QQué dificultades se me presentan para poder hacer cambios y mejorar? 
- Idea central: Se les explica sobre el tema de nuestra sesión, ¿Qué es el autoconocimiento? y se reflexionará sobre la frase puesta en el ppt (15 min). $\mathrm{Si}$ los estudiantes tienen preguntas, dialogar con ellos para cerrar la explicación.

- Cierre: Se les pide que peguen en el cuaderno su hoja de Bingo. Se les agradece por su participación y colaboración.

\section{IDEAS A TRANSMITIR}

- El conocerte, te va a permitir identificar tu propia forma de ser, tanto lo positivo como lo que uno tiene que mejorar, tus cualidades fortalecidas como tus defectos. Al ser sincero contigo mismo y no "esconder" aquello que no nos gusta de nosotros, nos damos la oportunidad de mejorar, crecer, aprender, tanto en los aspectos buenos como en los que aún tenemos que modificar y esforzarnos un poco más.

- El aceptar cómo somos realmente, no es fácil, es de valientes; pero la satisfacción de crecer como persona y darte cuenta de lo positivo que es para ti mismo y los que te rodean es aún mayor.

- Sabes poner límites a los demás, te valoras y te quieres. No te dejas llevar por los otros. El conocerte en los diferentes aspectos como el físico, emocional, psicológico, social, entre otros, ayuda a saber qué es lo que quieres y qué es lo que no deseas para ti. Por ejemplo, cuando un grupo se te acerque a ofrecerte algo que realmente no deseas o sabes que te hace mal, a pesar de que te presionen no vas a caer, porque tienes la convicción de que eso no te suma como persona.

- A veces tenemos creencias que son limitantes para nosotros mismos, como el "no soy buena para los deportes", "no soy creativa", "siempre voy a ser así", "nunca voy a cambiar", etc. El pensar de esa manera nos limita y no nos damos la oportunidad de seguir mejorando con el tiempo o de encontrar nuevas herramientas que nos permitan crecer en aquellas actividades que nos cuestan. Si miramos hacia atrás, nos daremos cuenta que no somos las mismas personas de hace 5 años, eso significa que he logrado avanzar y aprender de muchas cosas y lo tengo que tener presente en mi autoconocimiento.

\section{MATERIALES}

- Hoja de Bingo (pegarán en el cuaderno)

- Palabras y una bolsa (Bingo - Anexo 1)

- Premio para alumnos

- Pizarra

- Plumón de pizarra 


\section{APÉNDICE 4: EJEMPLO DE PRESENTACIÓN EN POWER POINT}

Con el permiso de la institución educativa, se presenta un ejemplo de las presentaciones en Power Point. La siguiente presentación es de la Sesión 3.
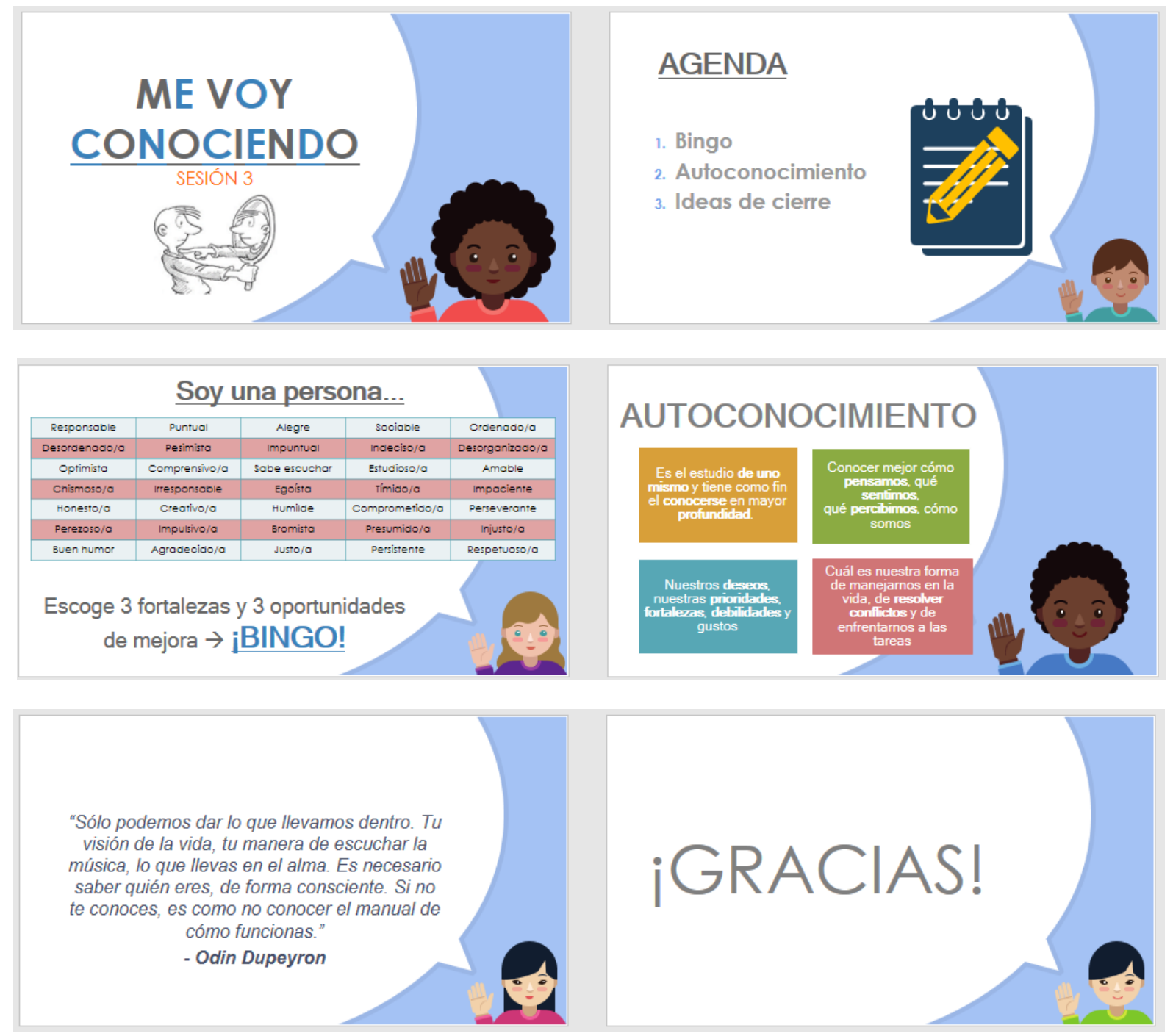